found to be a higher risk area for RTAs than towns, cities or suburban locations. Cats that were reported by their owners to hunt at the roadside were found to be at a greater risk of an RTA, as well as cats whose owners classified the stretch of road just by their house as being a 'long straight section of road'. No significant associations were found between coat colour, breed, sex or neuter status and risk of an RTA. Results from the study may help owners and rehoming centres to more accurately assess the risk of an RTA and may also facilitate better management to reduce the risk of an RTA.

\section{Road Traffic Accidents in cats attending emergency-care practices in the UK.}

\section{Megan Conroy', Dan O'Neill', Amanda Boag', David Church', David Brodbelt ${ }^{1}$}

1 Royal Veterinary College, Hatfield, UK

2 VetsNow Ltd, Dumfermline, UK

\section{BACKGROUND}

Road traffic accidents (RTA) are a common veterinary presentation and are reportedly the most common cause of mortality in young cats. However, there are limited publications describing risk factors and survival for RTA. This study aimed to estimate the prevalence of RTA in cats attending emergency out-of-hours clinics and to identify risk factors for RTA occurrence and survival to discharge.

\section{METHODS}

Using the VetCompass database, RTA cases were identified from the electronic patient records of cats attending Vets Now emergency clinics between January 2012 and February 2014. Mixed effects logistic regression was used to identify risk factors for RTA occurrence. Logistic regression was used to identify risk factors associated with survival to discharge.

\section{RESULTS}

There were 1,407 RTA cases identified from 33,053 cats attending 50 emergency clinics during the study period, resulting in an apparent prevalence of $4.3 \%$ (95\% Confidence Interval (C.I) 4.0\%-4.5\%). Cats aged 6months-2years had increased odds of RTA in comparison to all other ages (Odds Ratio (OR) range $0.14-0.67$ for all other ages, $\mathrm{p}<0.0001)$. Purebred cats (OR 0.53 95\% C.I 0.40-0.69 p<0.001) and female cats (OR 0.79 95\% C.I 0.75-0.96 p<0.001) had lower odds of RTA compared with crossbred and male cats respectively. A slight seasonal trend was present, with higher odds of RTA in the autumn (OR 1.19 95\% C.I 1.01-1.40 $\mathrm{p}<0.0001$ ) in comparison with the spring. Spinal (OR 2.44 95\% C.I 1.41-4.25 p=0.002) and abdominal injury (OR 2.71 $95 \%$ C.I 1.32-5.53 p=0.006) were associated with increased odds of death prior to discharge, and skin injury was associated with reduced odds (OR 0.27 95\% C.I 0.16-0.46 p<0.001) in comparison with cats without those injury types. Increasing count of injuries were associated with increased odds of death before discharge (OR 1.6 95\% C.I 1.29-1.98 p<0.001).

\section{CONCLUSION}

About one in 25 cats presenting to emergency-care practice have experienced an RTA event. Younger cats, males, and crossbreeds are at increased odds of RTA. The body-area injured and the count of injuries were associated with survival to discharge. Awareness of these risks may aid veterinarians to predict the risk of RTA for owners and to improve prognostic estimates for recovery.

\section{Attitudes to and opinions of neutering in dogs: results of a canine reproduction survey of veterinary surgeons}

\author{
Vicki Adams', Sarah Walker², Chris Taylor ${ }^{2}$ \\ 1 Vet Epi, Norfolk, UK \\ 2 Virbac, Suffolk, UK
}

An on-line survey was conducted to gather information about canine neutering. Invitations were placed on AnimalOracle.com, e-mailed to vetsurgeon.org members and sent via press releases. Questionnaire items were attitude statements with 5 answer options (Strongly agree, Agree, Disagree, Strongly disagree, No opinion) asking veterinary surgeons (VS) about perceptions and options to prevent breeding and sexual behaviours in dogs; most were asked on separate pages and respondents could not return to a question once they had moved on. A total of $411 \mathrm{VS}$ completed the survey: $71 \%$ were female, two-thirds were $<40$ years of age. Most recommended neutering of males and females although there was awareness of various alternatives to surgical neutering: $80 \%$ named Alizin to treat misalliance; 33\% Suprelorin for temporary medical castration and 27\% named Ypozane to treat BPH.

\title{
Question:
}

Do you recommend neutering as a routine procedure for all dogs?

Do you always recommend surgical neutering for female dogs not intended for breeding? male...?

Do you consider the various options available to owners when recommending methods to prevent unwanted puppies?

Do you offer owners alternatives to surgical neutering?

\begin{tabular}{|l|l|l|l|}
\hline Yes & \multicolumn{2}{|l|}{ No } \\
\hline N & $(\%)$ & N & $(\%)$ \\
\hline 310 & $(76)$ & 100 & $(24)$ \\
\hline 386 & $(94)$ & 24 & $(6)$ \\
\hline 251 & $(62)$ & 157 & $(38)$ \\
\hline 248 & $(60)$ & 162 & $(40)$ \\
\hline 211 & $(52)$ & 198 & $(48)$ \\
\hline
\end{tabular}




\section{Oral presentations}

\begin{tabular}{|c|c|c|c|c|c|c|c|c|}
\hline \multirow[t]{3}{*}{ Statement } & \multicolumn{2}{|c|}{$\begin{array}{l}\text { Strongly } \\
\text { Disagree }\end{array}$} & \multicolumn{2}{|c|}{ Disagree } & \multicolumn{2}{|c|}{ Agree } & \multicolumn{2}{|c|}{$\begin{array}{l}\text { Strongly } \\
\text { Agree }\end{array}$} \\
\hline & \multicolumn{2}{|l|}{1} & \multicolumn{2}{|l|}{2} & 3 & & 4 & \\
\hline & ${ }^{*} \mathbf{N}$ & $(\%)$ & $\mathbf{N}$ & $(\%)$ & $\mathbf{N}$ & $(\%)$ & $\mathbf{N}$ & $(\%)$ \\
\hline $\begin{array}{l}\text { I believe that all male dogs should be castrated if they are not going } \\
\text { to be used for breeding }\end{array}$ & 33 & (8.1) & 131 & (32.0) & 141 & (34.5) & 77 & (18.8) \\
\hline$\ldots \ldots$ female $\ldots$ spayed $\ldots$ & 3 & (0.8) & 18 & $(4.6)$ & 123 & (31.2) & 247 & (62.7) \\
\hline $\begin{array}{l}\ldots . . . \text { female } \ldots \text { spayed } \ldots \\
\ldots \text { before their third heat cycle } \ldots\end{array}$ & 8 & (2.0) & 36 & (9.1) & 135 & $(34.0)$ & 207 & (52.1) \\
\hline$\ldots \ldots$ second $\ldots$ & 8 & (2.0) & 40 & (10.1) & 146 & $(36.8)$ & 189 & (47.6) \\
\hline$\ldots \ldots$ first $\ldots$, around 5 to 6 months of age, ... & 26 & (6.6) & 109 & $(27.5)$ & 136 & (34.3) & 92 & (23.2) \\
\hline $\begin{array}{l}\text { I believe in early spaying for all female dogs if they are not going to } \\
\text { be used for breeding }\end{array}$ & 26 & (6.9) & 105 & (27.8) & 138 & $(36.5)$ & 80 & (21.2) \\
\hline $\begin{array}{l}\text { I believe that spaying too early may stunt a female dog's growth (i.e. } \\
\text { result in physical immaturity) }\end{array}$ & 11 & (2.8) & 117 & $(29.8)$ & 191 & $(48.6)$ & 28 & (7.12) \\
\hline
\end{tabular}

${ }^{\star}$ No opinion responses not shown above.

\section{A new approach proposal to canine reproductive cycle stage determination}

\section{Öge Gözütok}

University of Adnan Menderes, Aydin, Turkey

Macroscopic evaluation of the vaginal canal is one of the classical methods in determination of reproductive cycle stage of a canine patient. The main focus of this study is to develop a new and practical method, which is quicker and suited for a larger practitioner base in reaching the conclusive results than classical methods, which require more professional and possibly slower approaches. In the planned approach, the images are collected from the vaginal canal and the cervix through a vaginoscope and analyzed with a software developed specifically for this task. Outcome of the analysis is estimating the correct cycle stage within acceptable accuracy and reject unsuccessful samples. Through the literature survey, the structure of the study has been planned as collection of images from at least 30 different patients for each reproductive stage which will be labelled by classically approved methods; vaginal smears and measuring hormone levels. The classification model involves segmentation of images in the HSV color space, extraction of certain texture features such as contrast, correlation, energy and homogeneity through gray level co-occurrence matrix (GLCM) and training the Bayesian classifier with cross-validation. The outcome of this classification model is going to be, without any expert intervention, identification of the reproductive cycle stage of the canine patient from the collected images. The statistical texture analysis methods are successfully applied in other areas such as endoscopic analysis and oncological analysis. This work is a pioneer in applying those techniques to veterinary reproductive medicine. Therefore, this study is predicted to be useful for general practitioners as a practical and a less complicated method for cyclic stage determination, which will help maintaining the differential diagnosis list and estimate ovulation time.

\section{The prevalence of collapse in labradors in the uk and its association with exercise \\ Amber Mosley, Nick Bexfield, Mark Dunning}

School of Veterinary Medicine and Science, University of Nottingham, Nottingham, UK

Exercise induced collapse (EIC) is recognised in Labrador Retrievers (LR) and can lead to muscle weakness and collapse in otherwise healthy dogs. In working LR this can have a negative impact on their utility. The prevalence of EIC in UK $\mathrm{LR}$ has not been reported to date. A genetic test for mutated
Dynamin I (DNM1) is available for LR, a mutation that has been associated with EIC in the breed.

The aims of this study were to investigate the prevalence of collapse in UK Labradors, focusing on characteristics of the episodes, particularly whether this was associated with exercise. An additional aim was to determine how many of the dogs had been tested for the DNM1 mutation and associate this with the prevalence and characteristics of the collapse.

A questionnaire was designed and distributed to owners of UK LR, facilitated via the breed health groups. Questions included those specifically relating to the episodes of collapse (e.g. particular characteristics and whether this was at exercise), age of onset, general lifestyle factors and whether dogs had been tested for EIC.

684 questionnaires were returned, of which 88 (13\%) LR 